\title{
ON A GENERAL CLASS OF SECOND-ORDER, LINEAR, ORDINARY DIFFERENTIAL EQUATIONS SOLVABLE AS A SYSTEM OF FIRST-ORDER EQUATIONS
}

\author{
ROMEO PASCONE
}

\begin{abstract}
An approach for solving general second-order, linear, variable-coefficient ordinary differential equations in standard form under initial-value conditions is presented for the case of a specific constant-form relation between the two otherwise arbitrary coefficients. The resulting system of linear equations produces fundamental (or state transition) matrix elements used to create integral- and closed-form solutions for both homogeneous and nonhomogeneous differential equation variants. Two example equations are chosen to illustrate application. A short discussion is presented on the comparison of the theoretical results for these examples with the corresponding symbolic integration outputs provided by several commercial programs which were seen, at times, to be long and unwieldy or even non-existent.
\end{abstract}

Mathematics subject classification (2010): 34A30, 93C15.

Keywords and phrases: ordinary differential equations, systems of linear equations, fundamental matrices, nonhomogeneous systems.

\section{REFERENCES}

[1] G. Arfken And H. Weber, Mathematical Methods for Physicists, 6th Ed., Elsevier Academic Press, Burlington, MA, 2005.

[2] D. BADANI, On closed-form solutions to a class of ordinary differential equations, International Journal of Advanced Mathematical Sciences, 2, 1, (2014), 57-70.

[3] W. Boyce And R. Diprima, Elementary Differential Equations and Boundary Value Problems, 2nd $E d$., John Wiley and Sons, Inc., New York, NY, 1969.

[4] E. Coddington And N. Levinson, Theory of Ordinary Differential Equations, McGraw-Hill Book Company, New York, NY, 1955.

[5] V. P. ERmakov, Second-order differential equations: conditions of complete integrability, Applicable Analysis and Discrete Mathematics 2 (2008), 123-145.

[6] E. Garcia, L. Littlejohn, J.L. Lopez, E.P. Sinusia, Factorization of second-order linear differential equations and Liouville-Neumann expansions, Mathematical and Computer Modelling, 57, 5-6, (2013), 1514-1530.

[7] B. Goodwine, Engineering Differential Equations: Theory and Applications, Springer, 2011.

[8] HeunD-The Heun Doubleconfluent Function, http://www.maplesoft.com/support/help/Maple/view.aspx?path=HeunD, Maple Online Help. Maplesoft. Web. 7 Dec 2012

[9] F. John, Ordinary Differential Equations, Courant Institute of Mathematical Sciences, New York University, NY, 1965.

[10] W. Leighton, Ordinary Differential Equations, 3rd Ed., Wadsworth Publishing Company, Belmont, CA, 1970.

[11] G. Murphy Ordinary Differential Equations and Their Solutions, D. Van Nostrand Company, Princeton, NJ, 1960.

[12] P. SARAChiK, Principles of Linear Systems, Cambridge University Press, New York, NY, 1997. 
[13] M. SARAVI, A procedure for solving some second-order linear ordinary differential equations, Applied Mathematics Letters, 25, 3, (2012), 408-411.

[14] M TAYLOR, Introduction to Differential Equations, American Mathematical Society, Providence, RI, 2001.

[15] D. Zwilinger Handbook of Differential Equations, Academic Press, San Diego, CA, 1992. 